\title{
The experimental determination of unique green in the spectrum
}

\author{
LEO M. HURVICH, DOROTHEA JAMESON, AND JOSEPH D. COHEN \\ UNIVERSITY OF PENNSYLVANIA ${ }^{1,2}$
}

The notion is gaining currency that observers who are distributed normally with respect to commonly measured indices of color vision, may be bimodally distributed with respect to the spectral locus of unique green. Experimental conditions which can produce such deviations from a normal distribution are explored, and it is concluded that the "bimodality" results reported in the literature may reflect differential chromatic adaptation effects. Experimental conditions which guarantee a neutral state of adaptation yield spectral unique green loci that show no evidence of bimodality.

In a letter to Science about 15 years ago, concerning the issue of the "binocular fusion of yellow" (Hurvich \& Jameson, 1951a), Talbot (1952) proposed that the locus of unique green in the spectrum was bimodally distributed. As evidence for two classes of Os in an otherwise presumed "normal" population, he cited the data of Dimmick and Hubbard (1939). Of their group of $10 \mathrm{Os}$, Talbot pointed out that one class had a mean locus of unique green at $500 \mathrm{~nm}$, and another showed a mean of $525 \mathrm{~nm}$. He did, however, emphasize the need for more data on the issue.

Shortly after Talbot's letter appeared, the senior authors checked the spectral locus of unique green in a group of 25 normal Os for a neutral state of adaptation and found that the group formed a unimodal population with a mean at $501.7 \mathrm{~nm}$ and a total range that extended from $490.9 \mathrm{~nm}$ to $515.2 \mathrm{~nm}$. They did not publish the study but it will be discussed below in connection with a recent replication.

In the past few years, Talbot's suggestion has taken on a new lease of life. Rubin (1961), under Walls' guidance, tested 278 normals and determined the loci of the three unique spectral hues (blue, green, and yellow) and two binary hues (orange and cyan). He also made similar determinations on color defectives of various types. Here we are concerned only with the way the unique green locus is distributed in his normal sample and this distribution is shown in Fig. 1, a reproduction of Rubin's Fig. 1. Of his group, $68 \%$ found their unique green at loci that average to $514 \mathrm{~nm}$, and $32 \%$ at an average of $525 \mathrm{~nm}$.

Examination of Rubin's procedure makes it clear (1) that not all of his Os could have been in a neutral state of adaptation, and (2) that his particular experimental procedure for measuring the different unique hues leaves wide open the possibility of interserial adaptation effects. However valid the measures for the particular experimental conditions Rubin used, the "bimodality" reported was not for a normal population in a homogeneous, neutral equilibrium state of adaptation.

Linksz, in his engaging essay on color vision (1964), has now given this notion of the bimodality of the locus of unique green in a "normal" population potential theoretical importance. As a unifying concept, Linksz proposes that the spectral responses in the yellow-blue visual mechanism are probably bimodally distributed. Hence, the wavelength for unique green, which manifests itself at the transition point in the spectrum between the yellow and blue modes of response, must also be bimodally distributed in both normals and anomalous Os. ${ }^{3}$ Furthermore, a bimodality is also seen in the wavelength regions of spectral neutral points as determined in the two types of dichromats, namely, protanopes and deuteranopes. There are, however, serious discrepancies in spectral wavelengths for unique green and the neutral points among these three major groups (normal, anomalous, dichromatic). Rubin's green loci for normals, as noted, are at $514 \mathrm{~nm}$ and $525 \mathrm{~nm}$, for example, whereas the neutral points for the two different types of red-green blind dichromats are more nearly 495 and $500 \mathrm{~nm}$ (Walls \& Heath, 1956). Linksz recognizes these wavelength discrepancies, but deliberately limits himself to emphasizing the presumed duality of the yellow-blue transition locus for green (or neutral) in each of the three groups, independently of precise spectral positions.

In view of the increasing importance of the issue and the apparent discrepancy between our unpublished 1953 neutral adaptation data and Rubin's findings, we undertook to check the view that Rubin's particular experimental conditions and specific adaptational procedures are likely to yield a bimodal or at least skewed distribution of unique green measures in a normal population. Let us first briefly review his experiments.

In Rubin's experiments all of his Os were initially adapted to a $6 \mathrm{deg}$ field of $6500 \mathrm{deg} \mathrm{K}$ (Macbeth) illumination for $3 \mathrm{~min}$, at either $10 \mathrm{~mL}$ or $1 \mathrm{~mL}$ (the results are said to be independent of luminance level). One half of the $6500 \mathrm{deg} \mathrm{K}$ field was then extinguished and replaced by a monochromatic stimulus which the $\mathrm{O}$ adjusted so that its brightness matched the remaining half-field still illuminated by the $6500 \mathrm{deg} K$ light. While making the brightness adjustment, the $O$ simultaneously "bracketed" the continuously variable spectral light for the specific hue of interest, i.e., yellow, green, orange, etc. The $6500 \mathrm{deg} \mathrm{K}$ adapting half-field was then extinguished, and the wavelength setting was refined. Twelve determinations were made for each of five hues, yellow, green, cyan, orange, and blue, at the two luminance levels. It is especially important to note that the hue loci were obtained in precisely this same sequence for all Os, namely, yellow, green, cyan, orange, and blue. Each block of settings was preceded by $3 \mathrm{~min}$ of exposure to the adapting light, six measures were taken of each hue locus, and then the whole series was repeated in the same order. This order of measures is confirmed in a personal communication received from Rubin (1966). This procedure yielded the two average loci of unique green at $514 \mathrm{~nm}$ and $525 \mathrm{~nm}$, and a total range for all Os from $503 \mathrm{~nm}$ to $534 \mathrm{~nm}$.

Rubin's experimental procedures prompt three questions: (1) How does a $6500 \mathrm{deg} \mathrm{K}$ adapting field affect the distribution of unique green loci? (2) Since Rubin's hue loci were all determined to a single session, and since unique green judgments were always preceded by measurements of unique yellow loci, how is the determination of a unique green locus affected by preceding determinations of unique yellow loci? and (3) How are unique green settings affected if bracketing by the $O$ is not restricted to just-perceptibly bluish and yellowish limits? The same question

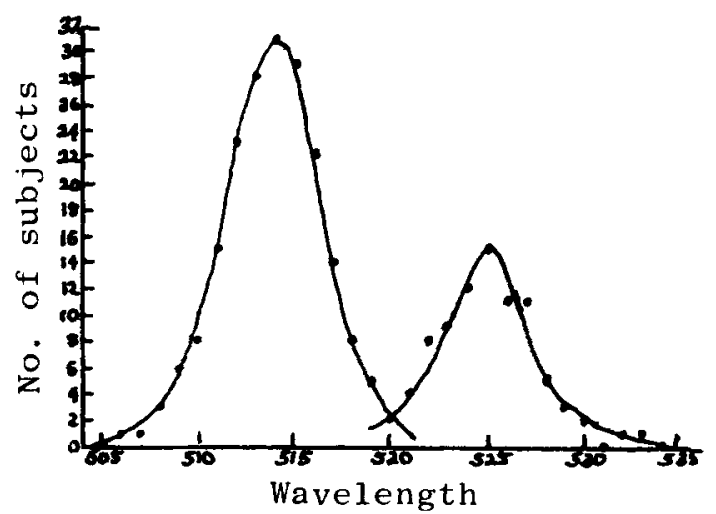

Fig. 1. Frequency distribution of unique green settings for 278 Os (from Rubin, 1961). 
can also be asked about the experimenter's "throw-off" of the wavelength drum between individual measurements. How does this affect the $O$ 's settings?

In a series of experiments directed toward answering these questions, the green unique hue locus was measured for a group of 23 normal Os composed largely of experimentally naive undergraduate students. The apparatus was that used in a color metric problem investigated by Krantz (1967) and apparatus details are described in his paper. A Bausch and Lomb $500 \mathrm{~mm}$ diffractiongrating monochromator was used to provide a 44' test field delimited on a ground glass diffuser. For the first set of measures, the surround was dark and the experiments were preceded by 10 min of light exclusion (neutral adaptation). The group was then retested after $3 \mathrm{~min}$ of bright adaptation to a $6500 \mathrm{deg} \mathrm{K}$ surround, $23 \mathrm{deg}$ in diameter, in which the $44^{\prime}$ test field aperture was centered. The surround remained illuminated throughout the remainder of the experimental session.

A method of adjustment was used and six measures were recorded for each adaptive condition. Brief intermittent exposures of the test field were made (approximately $5 \mathrm{sec}$ ). Each O's adjustment was made starting from a predetermined E's setting of the monochromator to one of a series of six different wavelength positions. Figure $2 \mathrm{~A}$ shows the wavelength displacements that occurred for the group between unique green settings in the dark surround and those for the $6500 \mathrm{deg} \mathrm{K}$ adapting field. The shifts to both longer (positive) and shorter (negative) wavelengths are
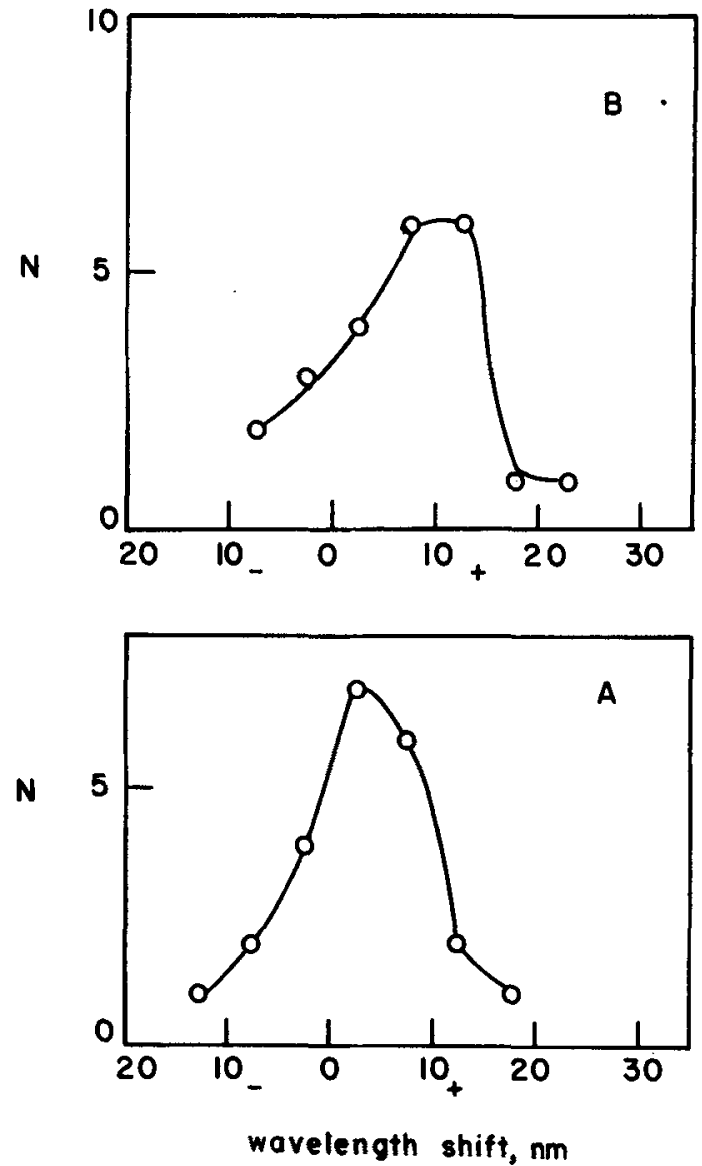

Fig. 2. (A) Distribution of wavelength displacements for the group of 23 Os from unique green settings in the dark surround to settings in the $6500 \mathrm{deg} K$ adapting field. (B) Distribution of wavelength displacements for the group of $23 \mathrm{Os}$ from unique green settings in the dark surround to settings in the 6500 deg $\mathrm{K}$ adapting field following interpolated sets of unique yellow measures.

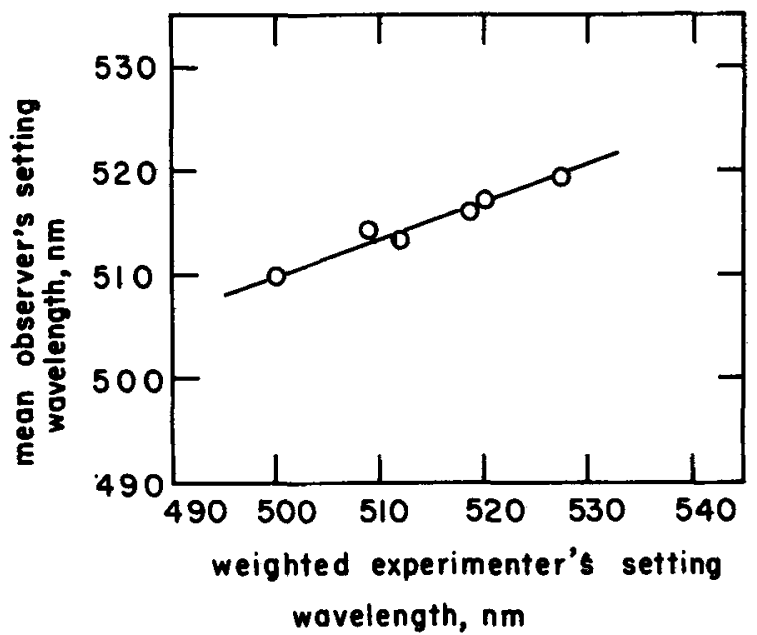

Fig. 3. Mean unique green setting for 21 observers in the dark surround as function of the weighted experimenter-determined starting point.

shown on the abscissa, the number of Os on the ordinate. Although about half of the Os gave essentially the same result for the $6500 \mathrm{deg} \mathrm{K}$ adaptive conditions as they did for the dark surround (a displacement toward longer, plus, or shorter, minus, wavelengths of $5 \mathrm{~nm}$ or less), the other half of the group showed changes that exceed $5 \mathrm{~nm}$. Moreover, we note that different $O$ s are differentially affected with regard to the direction of wavelength displacement for unique green between the two surround conditions. Clearly, $6500 \mathrm{deg} \mathrm{K}$ is not a chromatically-neutral light stimulus for all members of this group. This is not surprising when we consider some of the available data on the question of what light distributions are perceptually neutral (Hurvich \& Jameson, 1951 b; Jameson \& Hurvich, 1951; see also references there cited). It should also be noted that significant displacements occurred despite the fact that the luminance of the test field, which approximated $7.5 \mathrm{~mL}$ at $500 \mathrm{~nm}$, was greater than that of the surround which measured approximately $1.25 \mathrm{~mL}$. Simultaneous and successive inductive effects between surround (adapting) and test fields are known to decrease as the surround to test luminance ratio decreases (Hurvich \& Jameson, 1959). Hence, it seems very likely that inductive effects are greater in Rubin's experiments where the $6500 \mathrm{deg} \mathrm{K}$ adapting field was equal in luminance to the test fields. The measured unique green loci would seem to be "non-neutral" loci for some members of his population.

To check the effect on unique green settings of preceding yellow unique point settings, we proceeded as follows. After the six green measures for the $6500 \mathrm{deg} \mathrm{K}$ adaptation had been obtained, six measures of the unique yellow locus were determined. The green locus was redetermined, yet another set of yellow measures was repeated, and finally six more unique green measures were made. These measures were all obtained in the presence of the $6500 \mathrm{deg} K$ surround, and each block of settings was preceded by $3 \mathrm{~min}$ of exposure to the surround illumination, as in the Rubin procedure. The test exposures were continuous during adjustment, then the test field was briefly exposed again for a final check. What is the effect on the unique green locus of interpolating sets of unique yellow measurements? The wavelength shifts are shown in Fig. 2B. In contrast to the shifts produced by the $6500 \mathrm{deg} K$ adaptation treatment, for the majority of the group there was a displacement of the final set of unique green loci towards longer wavelengths following the additional series of yellow. green, and yellow determinations. The comparison in both cases is, of course. witl the green locus for the same Os in the initial, dark-surround condition. The skewness in the direction of larger positive difference is greater than in Fig. 2A. Fewer Os now show minor displacements $(0$ to $5 \mathrm{~nm})$, compared with those produced by the simple $6500 \mathrm{deg} K$ adaptation. But of more interest, is related to Rubin's "bimodality" result, is the fact that 
Table 1

Unique Green Loci

(Neutral Adaptation)

\begin{tabular}{|c|c|c|c|}
\hline Observer & Locus $\pm \sigma$ & Observer & Locus $\pm \sigma$ \\
\hline R. B. & $490.9 \pm 0.68$ & *A. B. & $502.6 \pm 0.92$ \\
\hline F. L. & $492.1 \pm 2.74$ & J. R. & $502.6 \pm 1.48$ \\
\hline *J. J. & $492.1 \pm 0.80$ & *B. H. & $503.0 \pm 1.26$ \\
\hline L. H. & $492.2 \pm 0.74$ & G. G. & $503.0 \pm 1.27$ \\
\hline R. C. & $492.8 \pm 0.93$ & *N. G. & $503.6 \pm 0.58$ \\
\hline G. Z. & $495.3 \pm 1.33$ & W. S. & $504.0 \pm 1.17$ \\
\hline W. F. & $496.7 \pm 1.19$ & *D. A. & $505.0 \pm 0.71$ \\
\hline *T. W. & $498.8 \pm 2.04$ & J. F. & $505.4 \pm 1.74$ \\
\hline R. B. & $498.8 \pm 1.23$ & K. L. & $505.4 \pm 1.99$ \\
\hline J. D. & $498.8 \pm 0.93$ & *A. M. & $505.4 \pm 0.97$ \\
\hline *J. C. & $499.0 \pm 0.45$ & H. B. & $505.5 \pm 1.00$ \\
\hline *A. K. & $499.0 \pm 1.26$ & S.P. & $506.7 \pm 1.54$ \\
\hline J. R. & $499.0 \pm 2.00$ & C. R. & $506.9 \pm 1.34$ \\
\hline I. Z. & $499.1 \pm 0.77$ & $* C . G$ & $507.0 \pm 1.89$ \\
\hline${ }^{*} \mathrm{E}, \mathrm{T}$. & $499.3 \pm 2.79$ & *M. S. & $507.1 \pm 0.80$ \\
\hline$* \mathbf{J} . \mathrm{C}$. & $499.5 \pm 0.55$ & E. S. & $507.5 \pm 2.00$ \\
\hline *S. M. & $500.1 \pm 1.53$ & ${ }^{*} \mathrm{C} . \mathrm{R}$. & $509.2 \pm 1.17$ \\
\hline R. 0 . & $500.4 \pm 2.54$ & R. D. & $509.6 \pm 0.89$ \\
\hline *N. M. & $500.7 \pm 1.12$ & *D. W. & $510.3 \pm 2.44$ \\
\hline *C. A. & $500.7 \pm 2.32$ & *R. S. & $510.4 \pm 1.74$ \\
\hline D. H. & $500.7 \pm 0.84$ & *J. E. & $510.4 \pm 0.73$ \\
\hline *J. F. & $501.0 \pm 0.77$ & *C.S. & $511.6 \pm 0.86$ \\
\hline R. S. & $501.1 \pm 0.66$ & ${ }^{*}$ H. S. & $514.2 \pm 1.47$ \\
\hline *B. C. & $501.4 \pm 1.20$ & J.P. & $515.1 \pm 2.04$ \\
\hline \multirow[t]{2}{*}{ D. H. } & $501.8 \pm 1.86$ & J. L. & $515.2 \pm 1.33$ \\
\hline & & Mean & $502.8 \pm 5.89$ \\
\hline
\end{tabular}

-1967 Observers

six Os show displacements toward longer wavelengths of 5 to $10 \mathrm{~nm}$, six show 10 to $15 \mathrm{~nm}$ shifts, and there is one each in the 15 to $20 \mathrm{~nm}$, and 20 to $25 \mathrm{~nm}$ ranges. Since the remaining Os are relatively unchanged or even move toward shorter wavelengths, it is clear that the serial effects, which seem to reveal differential adaptive responses to spectral light exposures, are indeed capable of distributing a group of Os over a wider range than prevails in the neutral state, and in an asymmetrical way. ${ }^{4}$

Evidence on our third question is available from examination of the effects on individual settings within a block of six trials. In the particular experimental procedure adopted, the wavelength drum of the monochromator was preset by the E for 21 of the $23 \mathrm{Os}$, and the E's sequence of settings was the same in all cases. The particular order was: $500,530,540,490,520$, and $510 \mathrm{~nm}$. A simple scatter plot of all Os' settings against the given sequence indicated that the E's setting exerted an influence. A more pertinent check of this point is made by assuming that chromatic light stimulation determined by each of the E's settings pools with that of his prior settings. Hence, a plot of the mean setting by the group as a function of the weighted, experimenter-determined, starting point was made. The weighting of the E's starting point is arbitrary and simply averages a given starting point with the mean of the previous experimenter settings. Thus the mean datum for a starting point at $500 \mathrm{~nm}$ is plotted at $500 \mathrm{~nm}$, that for a starting point of $530 \mathrm{~nm}$ is plotted at $515 \mathrm{~nm}=(500+530) / 2$, that for $540 \mathrm{~nm}$ plots at $527.5 \mathrm{~nm}=(515+540) / 2$, and so on.

The result is shown in Fig. 3. It is clear that the O's setting of unique green is determined to some extent by the E's initial settings, and thus by the spectral light stimulation immediately preceding the O's own wavelength adjustment.

The study described above was designed to mimic certain aspects of the Rubin experiments in order to explore the effects on unique hue determinations of specific procedures thought to influence the state of chromatic adaptation. Our results lead us to believe that a skewed, or bimodal distribution of the sort obtained by Rubin, although a perfectly valid finding for his conditions of measurement, is generated to a large extent by the specific adaptive conditions and experimental manipulations he employed.

Further support for this view comes from the 1953 experiment mentioned earlier and a recent (1967) replication of it. On both occasions, the locus of unique green in the spectrum was determined for $25 \mathrm{Os}$ with normal color vision. The earlier group was composed of members of the Color Technology Division of the Eastman Kodak Company, the second group of 25 normal Os were graduate students and staff members of the Psychology Department at the University of Pennsylvania.

The apparatus, procedure, instructions and experimenter were the same on both experimental occasions. Only the prevailing room illumination which was on during the preliminary familiarization period differed in the two instances. The room illumination was incandescent in the first experiment but fluorescent lights illuminated the room in the more recent experiment. In neither case was any general room illumination present either during the experiments proper or for $10 \mathrm{~min}$ prior to the first measurements.

To obtain these measurements, a monocular short focus telescope $(M=10 \mathrm{x})$ was focused on the exit slit of a Farrand prism monochromator so delimited that the $\mathrm{O}$ saw a foveal field $(0.8 \mathrm{deg} \times 1.3 \mathrm{deg})$ at a luminance level of about $25 \mathrm{~mL}$. Each $\mathrm{O}$ simply adjusted the wavelength drum of the monochromator until the test field he saw was a green that was neither yellowish nor bluish in appearance. The $E$ controlled the stimulus exposures which were repeated and brief ( 1 to $3 \mathrm{sec}$ duration), and the Os were instructed to bracket between a just perceptibly yellow and just perceptibly blue appearance and to stop when the field appeared neither yellowish nor bluish. Exposures were continued until the $O$ achieved the desired neither-yellowish-nor-bluish end-point. Individual means are based on five measures in all cases. The E's "throw-off" between repeated measures was minimal, about $5 \mathrm{~nm}$ from the O's last setting.

All these unique point loci are for a neutral state of adaptation. This is a chromatically balanced equilibrium condition. After the $O$ s had made a series of settings during a familiarization period with the room illumination on, the room lights were turned off

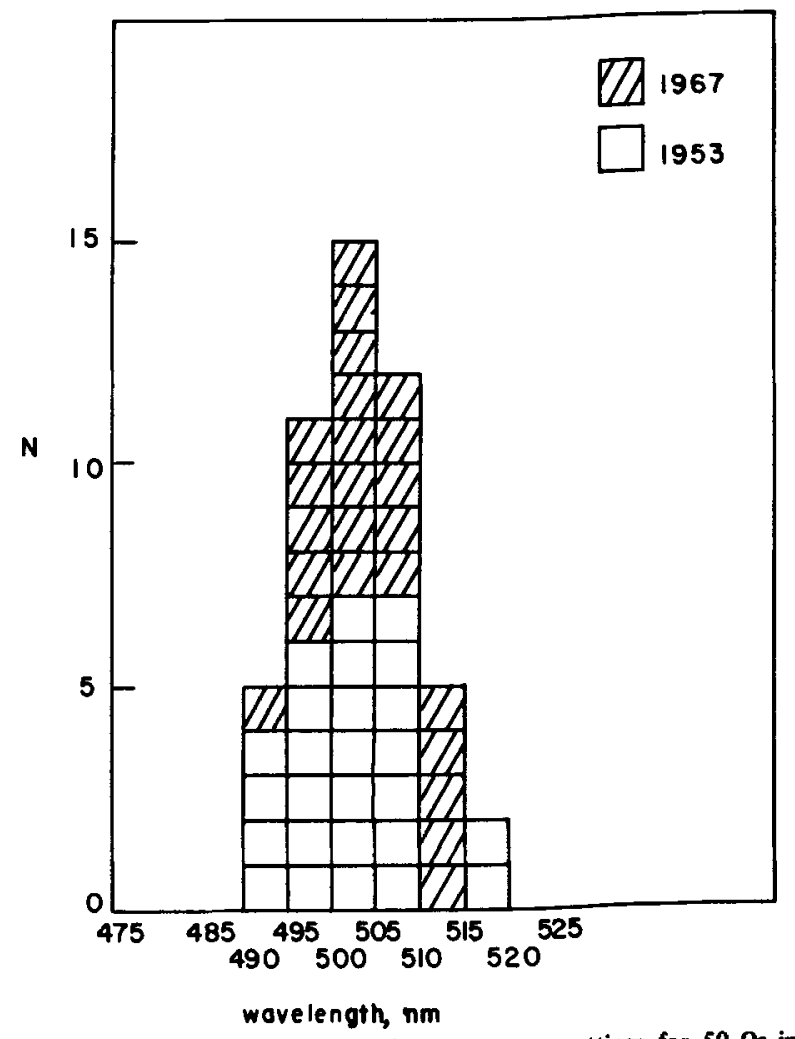

Fig. 4. Frequency distribution of unique green settings for $50 \mathrm{Os}$ in a neutral state of adaptation. 
and the $O$ sat in the dark for $10 \mathrm{~min}$. Ten minutes of light exclusion appears to be adequate to eliminate the effects of prior light stimulation which in general can be expected to produce a chromatically imbalanced state (Jameson \& Hurvich, 1951).

The data for the $50 \mathrm{Os}$, separately coded by years, are given in Table 1. As noted earlier, the mean for the 1953 measures is 501.7 nm. The data for the 1967 group range from $492.1 \mathrm{~nm}$ to $514 \mathrm{~nm}$ and the group mean is $503.7 \pm 5.11 \mathrm{~nm}$. Figure 4 summarizes graphically the data for the total population of 50 . There is little question that these $50 \mathrm{Os}$, measured 14 years apart in two groups of 25 , form a single population. The mean occurs at $502.8 \mathrm{~nm} \pm$ 5.89 and the total range of green loci extends from $490.9 \mathrm{~nm}$ to $515.2 \mathrm{~nm}$.

There remains an interesting problem that merits further exploration, and that is the different susceptibility of different individuals to changes in adaptive conditions. That $6500 \mathrm{deg} \mathrm{K}$, a broad band stimulus, produces differential effects compared to the neutral state is probably not surprising. Given individual differences in ocular transmission and density of macular pigment, $6500 \mathrm{deg} \mathrm{K}$ is effectively a different stimulus for each $\mathrm{O}$. But what is there about the visual system that causes different $O$ s to be differentially affected by what is effectively the same spectral stimulus, i.e., that stimulus which appears to be a unique yellow?

\section{REFERENCES}

DIMMICK, F. L., \& HUBBARD, M. R. The spectral location of psychologically unique yellow, green, and blue. Amer. J. Psychol, 1939, $52,242-254$.

HURVICH, L. M., \& JAMESON, D. The binocular fusion of yellow in relation to color theories. Science, 1951 a, 114, 199-202.

HURVICH, L. M., \& JAMESON, D. A psychophysical study of white. I. Neutral adaptation. J. opt. Soc. Amer., 1951b, 41, 521-527.

JACOBS, G. H., \& WASCHER, T. C. Bezold-Bruecke hue shift: further measurements. J. opt. Soc. Amer., 1967, 57, 1155-1156.

JAMESON, D., \& HURVICH, L. M. Use of spectral hue-invariant loci for the specification of white stimuli. J. exp. Psychol., 1951, 41, 455-463.

JAMESON, D., \& HURVICH, L. M. Perceived color and its dependence on focal, surrounding, and preceding stimulus variables. J. opt. Soc. Amer., $1959,49,890-898$.

KRANTZ, D. H. Small-step and large-step color differences for monochromatic stimuli of constant brightness. J. opt. Soc: Amer., 1967, $57,13041316$.

LINKSZ, A. An essay on color vision and clinical color-vision tests New York: Grune and Stratton, 1964.

RICHARDS, W. Differences among color normals: classes I and II. J. opt. Soc. Amer., 1967, 57, 1047-1055.

RUBIN, M. L. Spectral hue loci of normal and anomalous trichromats. Amer. J. Ophthalmol, 1961, 52, 166-172.

RUBIN, M. L. Personal communication, 1966.

TALBOT, S. A. Green vision and binocular fusion of yellow. Science, 1952, $115,220$.

WALLS, G. L., \& HEATH, G. G. Neutral points in 138 protanopes and deuteranopes. J. opt. Soc. Amer., 1956, 46, 640-649.

\section{NOTES}

1. This study was supported by a research grant, No. NB-04342, from the National Institutes of Health.

2. Address: Department of Psychology, University of Pennsylvania, Philadelphia, Pa. 19104

3. Linksz believes that both the red-green and yellow-blue systems are probably bimodally distributed.

4. Two recent additions to the unique green locus liferature tend to support a "bimodality" effect (Richards, 1967; Jacobs \& Wascher, 1967). Descriptions of the experimental procedures in both instances make it clear, however, that adaptation could not have been neutral for all Os and hence we see little point in further analyzing these studies.

(Accepted for publication February 28, 1968.) 